\title{
Fibromialgia e Reação Hansênica
}

\section{Fibromyalgia and Leprosy Reaction}

\author{
Sueli Coelho da Silva Carneiro ${ }^{(1)}$
}

\section{HANSENÍASE}

A hanseníase é uma doença infecto-contagiosa crônica, granulomatosa, causada pelo Mycobacterium leprae e encontrada apenas no ser humano, cujo contato é interpessoal e prolongado. Caracteriza-se por sinais e sintomas clínicos, dermatológicos, reumatológicos e neurológicos. Tais manifestações, relacionadas à resistência imunológica do indivíduo ao bacilo, variam entre as diferentes formas clínicas. A susceptibilidade à infecção relaciona-se com a diminuição ou ausência de resposta imunológica específica inata. Na população geral, $95 \%$ dos indivíduos apresentam resposta excelente, não sendo capazes de desenvolver a doença $^{(1)}$. Os doentes são classificados clinicamente de uma forma espectral, em dois pólos (formas estáveis) e três grupos intermediários (instáveis). No pólo tuberculóide (TT) é observada a melhor resposta imune entre os que adoecem, baciloscopia da linfa negativa e teste de Mitsuda positivo. O estudo histopatológico com coloração especial para os bacilos raramente evidencia a presença do mesmo. O pólo virchowiano (LL) é caracterizado por ter a pior resposta imunológica, com múltiplas lesões mal delimitadas, baciloscopia de linfa fortemente positiva e teste de Mitsuda negativo. A histopatologia mostra grande quantidade de bacilos formando globias. Os pacientes com características intermediárias entre os pólos, são subdivididos, de acordo com a sua semelhança clínica, imunológica e laboratorial com as formas estáveis, em borderline-tuberculóide (BT), borderline-borderline (BB) e borderline-virchowiano $(B L)^{(1-4)}$. Há ainda uma forma indeterminada, que pode preceder todas as outras variedades clínicas e quando presente, reflete estado inicial da moléstia.

As reações hansênicas são divididas em: Tipo 1 , também denominada reação reversa, é mediada por um mecanismo de hipersensibilidade tardia, do tipo celular. O quadro clínico é caracterizado por exacerbação de lesões pré-existentes, aparecimento de lesões novas, podendo ter ou não febre, edema de mãos e pés e queixa de dor nos nervos periféricos (ulnar, mediano, fibular comum, tibial posterior). Paralisias, amiotrofias e deformidades são ocorrências graves. Esse tipo de reação ocorre tanto nos pacientes paucibacilares quanto nos multibacilares ${ }^{(1,4)}$; o Tipo 2 decorre da presença de imunocomplexos antígeno-anticorpo nos tecidos, que podem ter sido formados in loco ou depositados após circularem. Clinicamente, se manifesta como nódulos subcutâneos eritematosos, dolorosos, mais palpáveis do que visíveis. O eritema nodoso hansênico aparece em surtos, cada nódulo dura de uma a duas semanas, mas outros podem aparecer. Pode ser acompanhado de febre alta, linfonodomegalias, mal-estar, hiporexia, mialgia, neuropatia, artralgias, edema de mãos, pés e antebraços ou por poliartrite franca, que compromete os joelhos, tornozelos e pequenas articulações das mãos. Durante a reação, o osso subcondral pode colapsar e causar destruição da articulação adjacente. Podem ocorrer ainda comprometimento ocular, renal, testicular ou de outros órgãos. Menos comumente, as lesões podem ser do tipo eritema multiforme, pustulosas, necrosantes ou tipo fenômeno de Lúcio. A reação tipo 2 ocorre apenas nos pacientes multibacilares. Laboratorialmente, encontra-se anemia, deficiência de ferro, velocidade de sedimentação das hemácias aumentada e hiperfibrinogenemia. Albuminúria pode estar presente e alterações bioquímicas podem ser encontradas ${ }^{(5,6)}$. O tratamento é feito com $60 \mathrm{mg}$ de prednisona por dia. A dor, a sensibilidade e os sintomas gerais rapidamente regridem ainda que possam recorrer ${ }^{(5,6)}$.

Recentemente, vivenciamos três casos de pacientes do sexo feminino, na quinta década de vida, que tiveram

Recebido em 10/10/2005 Aprovado, após revisão, em 10/02/06

1. Professora adjunta de Dermatologia da Faculdade de Ciências Médicas e Professora do Curso de Pós-graduação da Universidade do Estado do Rio de Janeiro (FCM-UERJ); Professora do Curso de Pós-graduação em Dermatologia da Faculdade de Medicina da Universidade Federal do Rio de Janeiro (UFRJ); Responsável pelo ambulatório de doenças cutâneo-articulares do Hospital Universitário Clementino Fraga Filho da UFRJ; Pós-doutorado pela Universidade de São Paulo (USP); Membro titular da Academia Brasileira de Reumatologia.

Endereço para correspondência: Sueli Coelho da Silva Carneiro, Rua Farme de Amoedo, 140, apto.601, Ipanema, Rio de Janeiro, CEP22420-020, RJ, Brasil, telefone: (21) 2523-5881 / (21) 9606-4849, e-mail:sueli@hucff.ufrj.br 
hanseníase multibacilar e foram tratadas com esquema PQT-MB - OMS/MS. Durante e após o tratamento, apresentaram episódios de dor musculoesquelética em várias localizações, rigidez matinal, artralgias, fadiga, cefaléia, parestesias e alterações do sono (hipersonia e/ou insônia), além de eritema nodoso, mal-estar e febre, sintomas estes que podem caracterizar reação hansênica. Foram tratadas com prednisona na dose de $1 \mathrm{mg} / \mathrm{kg}$ de peso, obtendo resposta terapêutica satisfatória. $\mathrm{O}$ acompanhamento destas pacientes, no entanto, mostrou que muitas das queixas persistiram, principalmente as de dor difusa, fadiga, parestesias, artralgias vagas, cefaléias eventuais, e alterações do sono, mesmo após desaparecerem as evidências clínicas de reação hansênica.

A avaliação reumatológica clínica e laboratorial permitiram fazer o diagnóstico de fibromialgia (FM), confirmado pela presença de dor à palpação de pelo menos 11 de 18 tender points (critérios do American College of Rheumatology - ACR,1990, Tabela 1).

Tratadas com antiinflamatórios não-esteroidais, antidepressivos tricíclicos, analgésicos e atividade física programada, com exercícios aeróbicos e de alongamento, obtiveram melhora gradativa de seus sintomas (Quadro 1).

A hanseníase e as reações hansênicas podem mimetizar muitas doenças dermatológicas, reumatológicas e neurológicas. Em países com alta endemicidade como o Brasil, há tendência de se superdiagnosticar esta doença ${ }^{(7)}$. O sinal maior da hanseníase é a hipo ou anestesia, no entanto, há muitas doenças reumatológicas e neurológicas que apresentam alterações da sensibilidade. Neuropatia por compressão é vista em vários sítios por variações anatômicas, por doença profissional e por síndrome do túnel do carpo ou do túnel do tarso. Os sintomas são de parestesia, fraqueza muscular e dor irradiada ${ }^{(7)}$.

A FM é doença comum na clínica reumatológica, representando cerca de $5 \%$ das consultas ambulatoriais e de 15 a $20 \%$ daquelas que acontecem nos ambulatórios de reumatologia. Atinge principalmente as mulheres entre a quarta e quinta décadas de vida ${ }^{(8)}$.

Os episódios reacionais ocorrem nas formas borderline tanto tuberculóide quanto virchowiana, durante ou após o tratamento. Caracterizam-se por sintomas gerais, eritema nodoso, artralgias, mialgias, parestesias e rigidez articular (Tabela 1).

Os médicos, em geral, são treinados para diagnosticar e

QUADRO 1

CARACTERÍSTICAS DAS PACIENTES QUE TIVERAM OS DIAGNÓSTICOS DE REAÇÃO HANSÊNICA E FIBROMIALGIA

\begin{tabular}{|c|c|c|c|c|}
\hline $\begin{array}{c}\text { Mulher, } 48 \mathrm{a}, \mathrm{br}, \\
\text { do lar, RJ }\end{array}$ & HBV - PQT Multibacilar & $\begin{array}{l}\text { Eritema nodoso, fadiga, } \\
\text { artralgias, dor difusa, } \\
\text { parestesias, alteração } \\
\text { do sono }\end{array}$ & $\begin{array}{c}\text { Prednisona } 1 \mathrm{mg} / \mathrm{kg} \text { peso } \\
\text { Pentoxifilina } \\
1200 \mathrm{mg} / \mathrm{dia}\end{array}$ & $\begin{array}{l}\text { Antiinflamatórios não- } \\
\text { hormonais, analgésicos, } \\
\text { antidepressivos tricíclicos, } \\
\text { atividade física programada }\end{array}$ \\
\hline $\begin{array}{c}\text { Mulher, } 50 \text { a, pd, } \\
\text { do lar, RJ }\end{array}$ & HBT - PQT Multibacilar & $\begin{array}{l}\text { Neurite, artralgias, } \\
\text { dor difusa, fadiga, } \\
\text { perturbação do sono }\end{array}$ & Prednisona - 1mg/kg peso & $\begin{array}{l}\text { Antiinflamatórios não- } \\
\text { hormonais, analgésicos, } \\
\text { antidepressivos } \\
\text { tricíclicos,exercícios }\end{array}$ \\
\hline $\begin{array}{c}\text { Mulher, } 49 \text { a, br, } \\
\text { do lar, RJ }\end{array}$ & HBT - PQT Multibacilar & $\begin{array}{l}\text { Parestesias, artralgias, } \\
\text { dor difusa, rigidez } \\
\text { articular, fadiga, pontos } \\
\text { dolorosos }\end{array}$ & Prednisona - $1 \mathrm{mg} / \mathrm{kg}$ peso & $\begin{array}{c}\text { Antiinflamatórios não- } \\
\text { hormonais, analgésicos, } \\
\text { antidepressivos tricíclicos, } \\
\text { exercícios }\end{array}$ \\
\hline
\end{tabular}


tratar doenças que tenham achados objetivos claros e alterações laboratoriais. Quando encontram pacientes com queixas dolorosas de músculos e articulações, com poucos achados ao exame físico, têm dificuldade em abordar tais pacientes, fazer o diagnóstico e propor tratamento efetivo.

Nós discutimos a concomitância de FM e hanseníase, assinalando 1) FM é uma doença comum com mais de $20 \%$ de prevalência na clínica reumatológica $(80-90 \%$ são mulheres entre 40 e 60 anos); 2) a FM é um reumatismo de partes moles caracterizado por dor tendinosa e muscular, adinamia, parestesia e sintomas não-específicos; 3 ) é possível a confusão entre estas manifestações e aquelas das reações hansênicas; 4) ambas as doenças podem ocorrer concomitantemente, com sinais e sintomas superpostos ou mimetizadores; 5) cada uma deve ser tratada separadamente.
TABELA 1

SinAIS E SINTOMAS QUE CARACTERIZAM A FIBROMIALGIA (7-10) E OS EPISÓDIOS REACIONAIS

\begin{tabular}{lll}
\hline \multicolumn{1}{c|}{ FIBROMIALGIA } & \multicolumn{1}{c}{ EPISÓDIOS REACIONAIS } \\
\hline - Fadiga & - Fadiga \\
- Parestesias & - Parestesias \\
- Rigidez articular & - Rigidez articular \\
- Sensação de edema de partes moles & - Áreas de anestesia \\
- Distúrbios do sono & - Distúrbios do sono \\
- Artralgias & - Artralgias \\
- Cefaléia & - Sintomas gerais \\
- Dor difusa & - Dor difusa \\
- Síndrome do túnel do carpo & - Síndrome do túnel do carpo \\
- Pontos dolorosos & - Nódulos eritematosos dolorosos \\
\hline
\end{tabular}

\section{REFERÊNCIAS}

1. Job CK: Pathology of leprosy. In: Hastings RC, editor. Leprosy. $2^{\circ}$ ed. New York: Churchill Livingstone: 193-224, 1994.

2. Ridley DS, Joppling WH: Classification of Leprosy according to immunity. A five group system. Int J Lepr other Mycobact Dis 3: 255-73, 1966.

3. Dharmendra. Classification of leprosy. In: Hastings RC, editor. Leprosy. $2^{\circ}$ ed. New York: Churchill Livingstone: 179-90, 1994.

4. Avelleira JC, Azulay-Abulafia L, Azulay DR, Azulay RD: Micobacterioses-Hanseníase. In: Azulay RD, Azulay DR, editores. Dermatologia. $3^{\text {a }}$ ed. Rio de Janeiro: Guanabara Kogan: 223-33, 2004.

5. Ramos-e-Silva, Carneiro SCS: Cutaneous Vasculitis in Latin America. Clinics in Dermatology 17: 663-70, 1999.

6. Freitas TC, Fleury RN: Hematologic Profile of Leprosy Patients in Reactional Episode of Erythema Nodosum Leprosum. Hansen Int 21: 59-66, 1996.
7. Global leprosy situation 2004. Weekly Epidemol Record/WHO 80: 118-124, 2005.

8. Feldman D: Fibromialgia: In. Nestor Schor ed.Guias de Medicina Ambulatorial e Hospitalar UNIFESP- Sato E. Reumatologia. São Paulo. Ed. Manole: 33-7, 2004.

9. Yunus MB, Masi AT: Fibromyalgia, Restless Legs Syndrome, Periodic Limb Movement Disorder, and Psychogenic Pain. In McCarty DJ \& Koopman WJ. Arthritis and allied conditions. Philadelphia, Lea \& Febiger: 1383-405, 1993.

10. Silva LC, Abeu AC, Pelegrino OS, Coelho OS: O valor da contagem de pontos dolorosos no diagnóstico clínico da fibromialgia. Rev Bras Reumatol 37: 317-22, 1997.

11. Wolfe F, Smythe HA, Yunus MB, et al: The American College of Rheumatology 1990 criteria for the classification of fibromyalgia: report of Multicenter Criteria Committee. Arthritis Rheum 33: 160-172, 1990.

12. Alarcón GS, Lowe JK: Avances em el conocimiento de La Fibromialgia Al Umbral del Tercer Milenio. Boletim Comité Ibero-Americano de Reumatologia IX: 8-12, 1999. 